\title{
Analyst
}

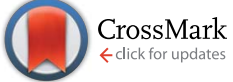

Cite this: Analyst, 2014, 139, 5362

Received 27th May 2014 Accepted 14th August 2014

DOI: $10.1039 / c 4 a n 00957 f$

www.rsc.org/analyst

\section{Green electrochemical sensing platforms: utilizing hydroxyapatite derived from natural fish scales as a novel electrochemical material for the sensitive detection of kidney injury molecule 1 (KIM-1) $\uparrow$}

\author{
Ying Zhang, ${ }^{a}$ Wei Zhang, ${ }^{\text {b }}$ Qing Zhang, ${ }^{c}$ Kaiyang Li, ${ }^{c}$ Wei Liu, ${ }^{* a}$ Yong Liu*c \\ and Craig E. Banks*d
}

\begin{abstract}
Urinary KIM-1 is an ideal biomarker for acute kidney injury diagnosis. The proof-of-concept is demonstrated by utilizing the hydroxyapatite derived from natural fish scales as an electrode material, where the sensing of KIM-1 is shown to be possible for the first time with a linear range from 0.01 to $0.20 \mu \mathrm{g} \mathrm{mL}^{-1}$ and a detection limit of $0.017 \mu \mathrm{g} \mathrm{mL}^{-1}$ under model conditions; proof-of-concept is demonstrated in spiked urine.
\end{abstract}

In recent years, acute kidney injury (AKI) is a very frequent and serious clinical problem, accounting for overall high morbidity and mortality. ${ }^{1}$ The up-regulation of KIM-1 is found in patients with renal cell carcinoma, urate nephropathy, acute and chronic tubular injury, allograft nephropathy and AKI after cardiac surgery. $^{2}$ As KIM-1 is not found in the urine of healthy people, its quantification can be used as an ideal biomarker for AKI. ${ }^{3}$ Currently, urinary KIM-1 measurement has been performed using ELISA assays or microsphere-based Luminex xMAP technology that requires a $3-4 \mathrm{~h}$ assay time, and is dependent on a large optical analyzer. ${ }^{4}$ Although the linearity, intra-run and inter-run precision, lower limit of quantification, recovery, dilutional verification, reference range, stability and length of run have been validated, cost-efficient, simple and convenient approaches are still required.

Electrochemical sensing technologies have been recognised as one of the most sensitive and facile methods for trace

${ }^{a}$ National Engineering Research Center for Nanomedicine, College of Life Science and Technology, Huazhong University of Science and Technology, Wuhan, 430074, P.R. China.E-mail: wliu@hust.edu.cn; Fax: +86-027-87792234; Tel: +86-027-87792234 ${ }^{b} 712$ th Research Institute, China Shipbuilding Industry Corporation, Wuhan, 430064, P.R. China

'State Key Laboratory of Powder Metallurgy, Central South University, Changsha, 410083, P.R. China. E-mail: yonliu@csu.edu.cn; Fax: +86-731-88836939; Tel: +86731-88836939

${ }^{d}$ Faculty of Science and Engineering, School of Science and the Environment, Division of Chemistry and Environmental Science, Manchester Metropolitan University, Chester Street, Manchester M1 5GD, Lancs, UK. E-mail: c.banks@mmu.ac.uk; Web: http:// www.craigbanksresearch.com; Fax: +44 (0)1612476831; Tel: +44 (0)1612471196

$\dagger$ Electronic supplementary information (ESI) available: See DOI: 10.1039/c4an00957f analysis of substances due to their favourable portability, suitability for automation, short analysis time, low power consumption and inexpensive equipment. ${ }^{5}$ However, there are very few reports about the electrochemical determination of AKI. In this field, only the indirect electrochemical immunosensor has been reported for the detection of neutrophil gelatinase-associated lipocalin (NGAL, a similar biomarker for AKI), which is developed by the immobilization of a rabbit polygonal lipocalin-2 antibody on gold nanoparticles attached on generation-1 polyamidoamine (PAMAM) dendrimer (LA2/AuNPs/ PAMAM)-modified gold electrodes. ${ }^{6}$ It is noted that to the best of the authors' knowledge, there are no electrochemical reports for the direct electrochemical sensing of KIM-1.

Electrochemists are always searching for new electrode materials for the improvement of electrochemical processes, particularly electro-analysis where improvements in the sensitivity and limit of detection can be realised. One approach is the use of what some might consider worthless materials, which can be transformed into useful resources. For example, in the field of electrochemistry, it has been demonstrated that fish scales can be transformed into a useful electrode material; hence the terminology "green electrochemistry" is used to indicate that the electrode material is derived from waste materials. Fish scales are considered worthless, impracticable, and usually dismissed as waste and each year, 18-30 million tons of fish waste is discarded throughout the world. ${ }^{7}$ However, it has been noticed that fish scales contain numerous valuable organic and inorganic components, mainly collagen and hydroxyapatite (HAp), which have a potential value for use in manufacturing functional foods, cosmetics, and biomedical products. ${ }^{7,8}$ In a similar fashion, it has been shown that HAp modified electrodes can be derived which have attracted much attention mainly attributed to their high biocompatibility and unique three-dimensional network in the lattice structure. ${ }^{9}$ In our previous research, HAp particles were synthesised by a template-assisted biomineralization strategy which were applied to the ultra-sensitive detection towards trace environmental pollutants, namely heavy metal ions, endocrine- 
disrupting chemicals, and toxic arsenides. ${ }^{\mathbf{1 0}}$ Additionally, it has been reported that the electron transfer process could be accelerated by HAp modified electrodes when used for the determination of significant biological substances, such as $\mathrm{L}^{-}$ tyrosine ${ }^{11}$ hemoglobin (Hb), ${ }^{12}$ and alpha fetoprotein (AFP) ${ }^{13}$ which displays much promising prospects in biomedical fields compared to carbon nanotubes (CNTs) or graphene modified electrodes. $^{\mathbf{1 4}}$

In this current communication, HAp extracted from fish scale waste after heating at different temperatures was utilized to fabricate a HAp modified carbon paste electrode (CMCPE) by mixing a certain amount of as-prepared HAp and graphite powder, and applied to the sensing of KIM-1. To the best of our knowledge, this is the first report of the electrochemical detection of this important biomarker; such an approach has the potential for the development of a new sensing protocol, which exhibits advantages such as high-sensitivity, portability, low cost and fast-responses. Typically, fish scales were collected from a black carp (a common freshwater fish) and treated by a two-step calcined procedure: $400{ }^{\circ} \mathrm{C}$ for 2 hours to remove almost all organic components at first, and then $700-1100{ }^{\circ} \mathrm{C}$ for 4 hours to obtain relatively pure HAp powders. For simplification, the HAp samples extracted at $700{ }^{\circ} \mathrm{C}, 900{ }^{\circ} \mathrm{C}$ and 1100 ${ }^{\circ} \mathrm{C}$ were separately marked as 700-HAp, 900-HAp and 1100-HAp; more information is given in the ESI. $\dagger$

Fig. 1 compares the X-ray diffraction (XRD) patterns of the HAp samples and shows distinct peaks of HAp $\left(\mathrm{Ca}_{5}\left(\mathrm{PO}_{4}\right)_{3} \mathrm{OH}\right.$, PDF 09-0432, main crystalline phase) with a small amount of whitlockite $\left(\mathrm{Ca}_{3}\left(\mathrm{PO}_{4}\right)_{2}\right.$, TCP, PDF 09-0169) or calcium phosphate hydrate $\left(\mathrm{Ca}_{3}\left(\mathrm{PO}_{3}\right)_{6} \cdot 10 \mathrm{H}_{2} \mathrm{O}\right.$, PDF 40-0008) found in all the samples. The crystalline parameters such as $2 \theta$, the interplanar spacing $(d)$ values for the strongest four diffraction peaks of (211), (300), (112) and (002) planes, and lattice constants in each calcined sample were respectively calculated by peak profile and cell refinement, in order to reflect the transformation tendency when increasing the calcination temperature, as shown in Table S1 of ESI. $\dagger$ Herein, only the values of $2 \theta_{211}, 2 \theta_{300}$ and $d_{112}$ increased with temperature, while the values of $2 \theta_{112}, d_{211}, d_{300}$,

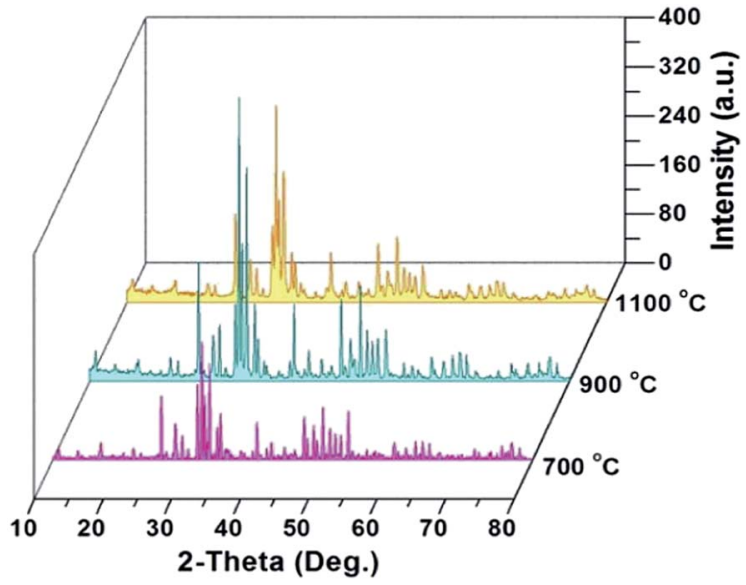

Fig. 1 XRD patterns of extracted HAp samples from fish waste after heating at 700,900 and $1100{ }^{\circ} \mathrm{C}$ for 4 hours, respectively. $d_{002}$ and XS (mean crystal size evaluated by the Scherrer equation) displayed a decrease. For the cell lattice, the variation of $a$, $c$ and $v$ with the temperature shows the cell transformation as schematically shown in Fig. 2. Consider that the electrochemical sensing capability mainly depends on the electron transfer process in the crystalline network of HAp, the variation of these lattice parameters will likely impact the sensing capability. A suitable crystalline lattice size will favour the electron transfer and enhance the electrochemical detection.

Corresponding scanning electron microscopy (SEM) images of HAp extracted from fish scale waste following thermal processing at $700-1100{ }^{\circ} \mathrm{C}$ show an elongated structure indicative of a native plywood structure (Fig. 3), which is similar to previous reports. ${ }^{15}$ It can be seen that the "strips" of calcium phosphate were separated by a spacing of 0.5-2 $\mu \mathrm{m}$. Each sheet appears to be structurally stabilized as an open structure by thin branchlike connections between the adjacent strips. No such connections were observed between adjacent sheets, which sometimes readily peeled away from the surface of the heated fibrillary plate. Moreover, the tendency that the size of apatite particles gradually increased with the heating temperature is clearly shown. It has also been noticed that the morphology of fish scales after heating at $1100{ }^{\circ} \mathrm{C}$ showed sintering and aggregation of the calcium phosphate crystallites to produce porous replicas with an interconnected granular texture. Typical domain sizes were 1-2 $\mu \mathrm{m}$, respectively. Small crystals of TCP with sizes of $0.1-0.2 \mu \mathrm{m}$ were also observed at the surface of this sample. The results suggested that the preferred crystallographic orientation of the apatite particles along the collagen fibres is highly replicated within the calcined product.

To investigate the sensing performances towards KIM-1, the HAp extracted from fish scales under different calcination temperatures were used to fabricate CMCPEs and explored in 0.2 M PBS. The electrochemical behaviours of the HAp modified CMCPEs towards the detection of $1 \mu \mathrm{g} \mathrm{mL}{ }^{-1}$ KIM-1 were investigated by linear sweep voltammetry (LSV) and cyclic voltammetry (CV) techniques. It is clear from the inspection of Fig. 4a that only background currents are observed at the pure carbon paste electrode (CPE), indicating its electro-inactive nature towards KIM-1. Meanwhile, no electrochemical reaction occurs on the CMCPE modified by $20 \%$ (w/w) of $900-H A p$ in 0.2 M PBS (blank). Fig. 4b indicates the effect of loading ratio (900HAp\%) upon the peak current $\left(i_{\mathrm{p}}\right)$ of KIM-1. The current density showed a tendency to increase at first, with the increasing amounts of modifier, because the concentration of HAp on the surface of the modified CPE increases correspondingly and

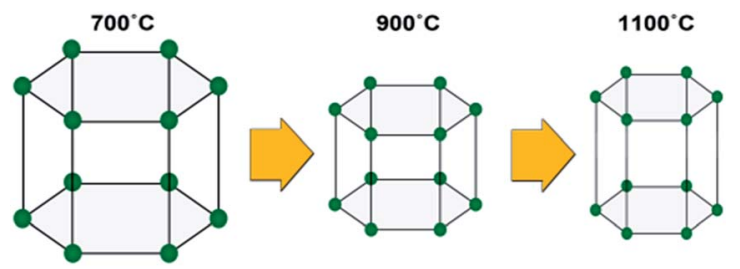

Fig. 2 Schematic of the cell transformation under different calcination temperatures of 700,900 and $1100{ }^{\circ} \mathrm{C}$. 

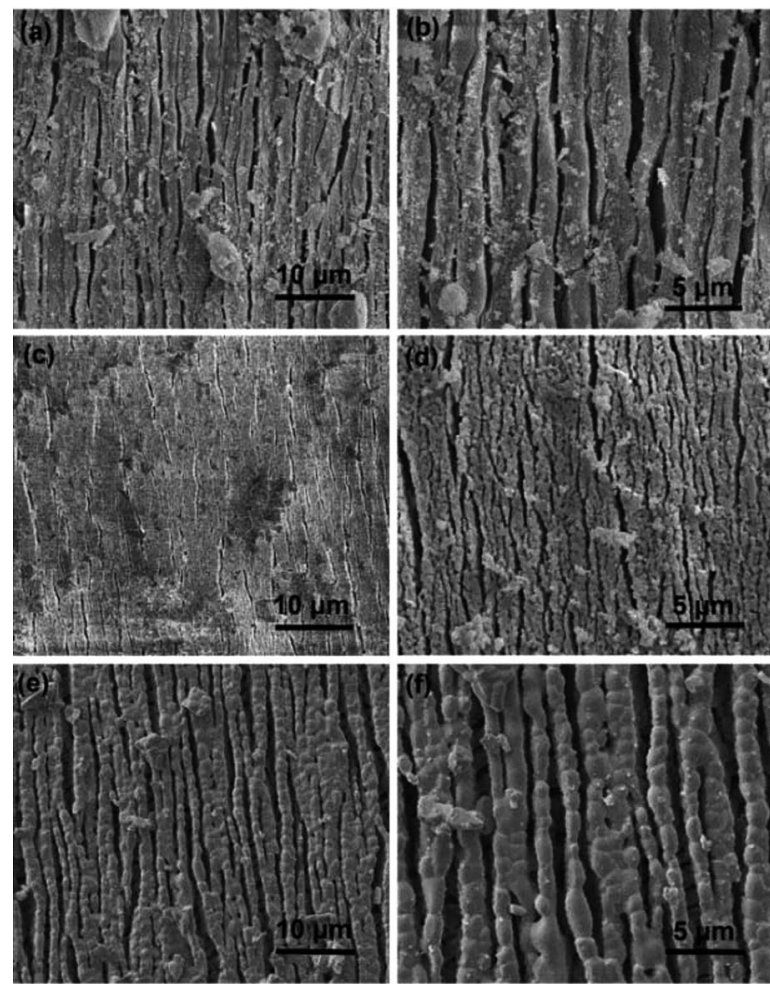

Fig. 3 Morphology characterization of each calcined sample at 700 ${ }^{\circ} \mathrm{C}$ ( $\mathrm{a}$ and b), $900{ }^{\circ} \mathrm{C}$ (c and d) and $1100{ }^{\circ} \mathrm{C}$ (e and f) for 4 hours.

likely supplies enough absorption sites for the target analyte. It is noted that for the electrode containing $20 \%$ of HAp, the highest $i_{\mathrm{p}}$ value was obtained. However, continuous increase of HAp loading results in a decrease of current response, possibly due to the fact that excessive HAp may result in the loss of conductive pathways through the modified electrode. Interestingly, a current decrease could be observed following successive
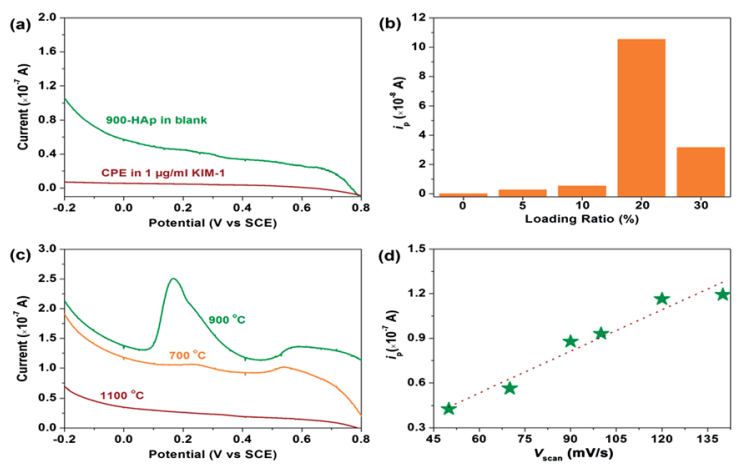

Fig. 4 Electrochemical behaviour of KIM-1 on CPEs and HAp modified CMCPEs. (a) Control groups for LSV curves of the CPE in $0.2 \mathrm{M}$ PBS containing $1 \mu \mathrm{g} \mathrm{mL}^{-1}$ of KIM-1, and the 900-HAp modified CMCPE in 0.2 M PBS without KIM-1 (blank). (b) Influence of loading ratio (900HAp\%) on the peak current $\left(i_{p}\right)$ measured by LSV. (c) LSV curves comparison for CMCPEs modified by HAp calcined at different temperatures. (d) The relationship between the peak current $\left(i_{p}\right)$ and the applied scan rate $\left(v_{\text {scan }}\right)$ measured by $C V$. The measurements of $(\mathrm{b}-\mathrm{d})$ were performed in $0.2 \mathrm{M}$ PBS containing $1 \mu \mathrm{g} \mathrm{mL} \mathrm{L}^{-1}$ of $\mathrm{KIM}-1$. measurements, which is likely a consequence of accumulation of the electro-oxidation products on the modified electrode surface. In order to overcome this, the electrode surface can be gently rubbed to provide a renewed surface - such an approach is common in the field of carbon paste electrodes. Moreover, by understanding the electrochemical behaviours of KIM-1 on CPEs modified by HAp extracted under different temperatures in Fig. 4c, it can be observed that the electrochemical oxidation of KIM-1 only occurs on a 900-HAp modified CPE, with a voltammetric peak observed at $\sim+0.2 \mathrm{~V}$ ( $v s$. SCE). This result may be associated with the lattice parameters of HAp where the 900HAp has the most suitable crystalline network among the three HAp samples, which is convenient for electron transfer/ adsorption processes. Furthermore, the dependence of $i_{\mathrm{p}}$ on the voltammetric scan rate $\left(v_{\text {scan }}\right)$ via $\mathrm{CV}$ measurement, as shown in Fig. $4 \mathrm{~d}$, shows that the $i_{\mathrm{p}}$ increases linearly with $v_{\text {scan }}$ in the range of $50-140 \mathrm{mV} \mathrm{s}^{-1}$, with an expression of: $i_{\mathrm{p}}\left(\times 10^{-7} \mathrm{~A}\right)=$ $0.0093 v_{\text {scan }}-0.023\left(R^{2}=0.94\right)$. This relationship reveals a typical adsorption-controlled process, which can be utilised quantitatively. Consequently, the calibration plot for the sensing of KIM-1 was investigated over the concentration range of $0.05 \mu \mathrm{g} \mathrm{mL}^{-1}$ to $0.8 \mu \mathrm{g} \mathrm{mL}{ }^{-1}$ via $\mathrm{LSV}$ measurements under the optimized working conditions, which exhibited a linear response over the range of $0.01 \mu \mathrm{g} \mathrm{mL}^{-1}$ to $0.20 \mu \mathrm{g} \mathrm{mL}^{-1}$ (Fig. 5). The relationship between $i_{\mathrm{p}}$ and the concentration of KIM-1 $\left(C_{\mathrm{KIM}-1}\right)$ can be expressed by the linear regression equation:

$$
i_{\mathrm{p}}\left(\times 10^{-7} \mathrm{~A}\right)=9.0503 C_{\mathrm{KIM}-1}\left(\mu \mathrm{g} \mathrm{mL}^{-1}\right)+0.2236\left(R^{2}=0.99\right) .
$$

Based on the above results, the detection limit $(3 \sigma)$ was estimated to correspond to $0.017 \mu \mathrm{g} \mathrm{mL} \mathrm{m}^{-1}$, indicating this proposed strategy can potentially be used to detect trace KIM-1 for AKI determination in future. Note that this is the first ever example of the direct electrochemical detection of KIM-1 and hence no direct comparison with any other electrochemical strategy is possible.

Next, to explore any potential false-positive or false-negative results that might be encountered when applied to real world analysis, the electrochemical protocol was applied to sense KIM-1 in real human urine. The human urine was collected (see

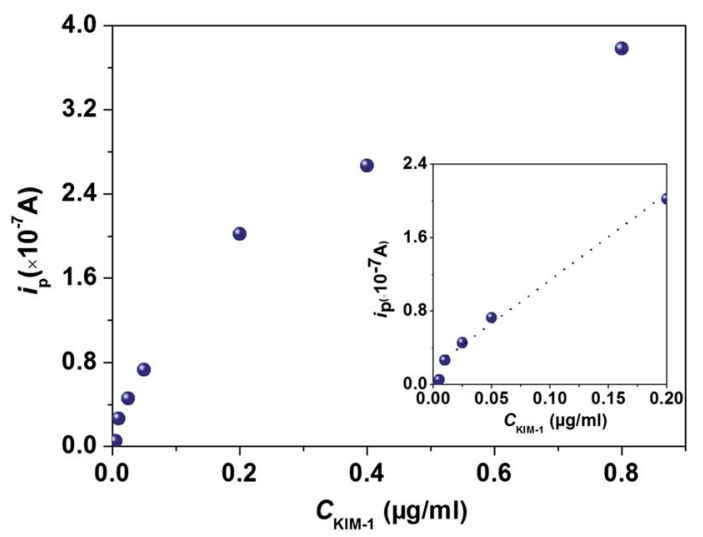

Fig. 5 Calibration plot of CMCPE modified by 900-HAp in 0.2 M PBS containing different concentrations of KIM-1. 
ESI $\dagger$ ) with the pH adjusted to 5.5 by diluting with $0.2 \mathrm{M}$ PBS (20 : 80; urine: 0.2 M PBS). Fig. 6 shows LSV signatures recorded in human urine and then in that spiked with KIM-1 where only an electrochemical signal is observed in the presence of KIM-1 indicating no interference when applied to real human urine; a large peak at around $+0.6 \mathrm{~V}$ is also observed, the origin of which is unknown but is well resolved from the analytical peak of interest.

The electrochemical protocol was repeated as described above (Fig. 5) with a human urine sample. A calibration plot was measured over the concentration range of $0.01 \mu \mathrm{g} \mathrm{mL}{ }^{-1}$ to 0.8 $\mu \mathrm{g} \mathrm{mL} \mathrm{m}^{-1}$ by square wave voltammetry (SWV) in order to increase the sensitivity of the electrochemical response in the real sample matrix.

Fig. 7 shows the corresponding calibration plot over the range of $0.01 \mu \mathrm{g} \mathrm{mL}^{-1}$ to $0.8 \mu \mathrm{g} \mathrm{mL}^{-1}$, where a linear response at concentrations of $0.01 \mu \mathrm{g} \mathrm{mL} \mathrm{L}^{-1}$ to $0.10 \mu \mathrm{g} \mathrm{mL}{ }^{-1}$ with sufficient reproducibility is observed in the inset. The linear regression equation is as follows:

$$
i_{\mathrm{p}}\left(\times 10^{-7} \mathrm{~A}\right)=20.795 C_{\mathrm{KIM}-1}\left(\mu \mathrm{g} \mathrm{mL}^{-1}\right)+1.0656\left(R^{2}=0.99\right) .
$$

In summary, we have shown the proof-of-concept of a green approach for the fabrication of a novel electrode material that facilities the electrochemical detection of KIM-1 in aqueous based solutions exhibiting a linear range of 0.01 to $0.20 \mu \mathrm{g} \mathrm{mL}^{-1}$ with a limit of detection corresponding to $0.017 \mu \mathrm{g} \mathrm{mL} \mathrm{m}^{-1}$ in model buffer solutions and is shown to be viable in spiked human urine with no interference. A commercially available immunoassay (RenaStick) has been evaluated towards the detection of KIM-1 in diluted urine reporting a limit of detection of $0.8 \mathrm{ng} \mathrm{mL}$ (ref. 16) which is lower than our observed value $\left(17 \mathrm{ng} \mathrm{mL}^{-1}\right)$ but note that the former requires $15 \mathrm{~min}$ for the test to develop.

Recently AKI has been explored in cytoreductive surgery where KIM-1 was measured at $143 \mathrm{ng} \mathrm{mL}^{-1}$ pre-operation increasing to $7834 \mathrm{ng} \mathrm{\textrm {mL } ^ { - 1 }}$ post operation (24 h) as measured by microbead based xMAP technology. ${ }^{16}$ Since elevations in urinary KIM-1 levels are monitored, the real challenge for our

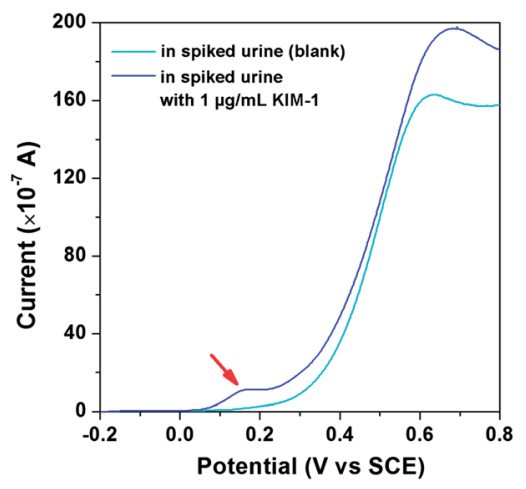

Fig. 6 LSV curves of the 900-HAp modified CMCPE in spiked urine containing $1 \mu \mathrm{g} \mathrm{mL}^{-1}$ of $\mathrm{KIM}-1$, and in a spiked urine sample without KIM-1 (blank).

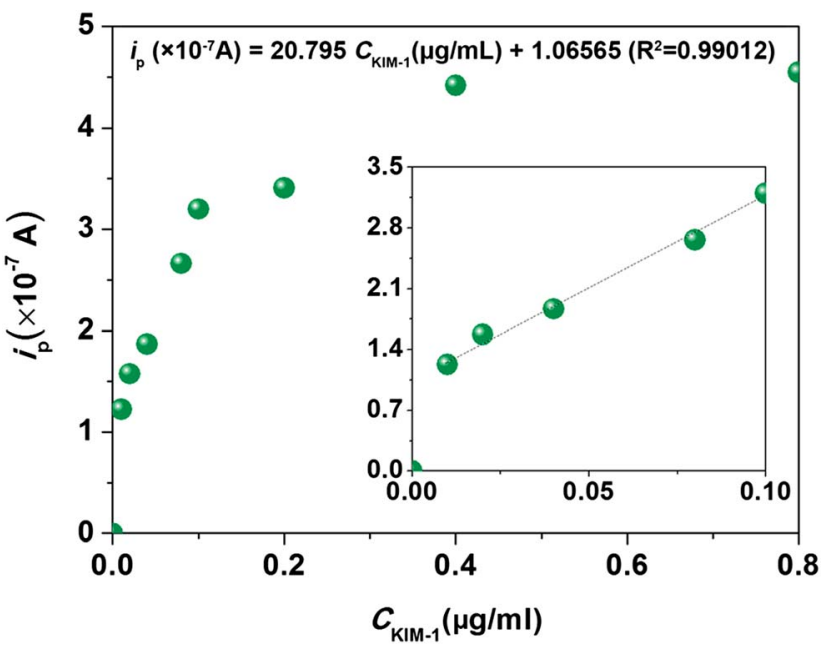

Fig. 7 Calibration plot of the CMCPE modified by 900-HAp in spiked urine samples containing different concentrations of KIM-1 using square-wave voltammetry using the following parameters: initial $E_{1}=$ $-0.2 \mathrm{~V}$; final $E_{2}=0.8 \mathrm{~V}$; increment $E=0.004 \mathrm{~V}$; amplitude $=0.025 \mathrm{~V}$; frequency $=15 \mathrm{~Hz}$.

protocol, and others being developed, is to be able to detect KIM-1 in urine at low levels encountered and also be able to distinguish its elevation allowing AKI to be medically diagnosed. This challenge is generic in nature to any test measuring KIM-1. Note that this is the first electrochemical report of the detection of KIM-1 and has the potential for the practical application for detecting KIM-1 in AKI patients' urine; such work is underway to try and overcome the challenges identified above.

\section{Acknowledgements}

This work was supported by Special Financial Grant and 2-class General Financial Grant from the China Postdoctoral Science Foundation (2013T60720 and 2012M521431), National High Technology Research and Development Program of China (863 Program, 2012AA022704), and National Natural Science Foundation of China (NSFC, 31170960).

\section{Notes and references}

1 A. Bagga, A. Bakkaloglu, P. Devarajan, R. Mehta, J. Kellum, S. Shah, B. Molitoris, C. Ronco, D. Warnock, M. Joannidis and A. Levin, Pediatr. Nephrol., 2007, 22, 1655; B. Molitoris, A. Levin, D. Warnock, M. Joannidis, R. Mehta, J. Kellum, C. Ronco and S. Shah, Nat. Clin. Pract. Nephrol., 2007, 3, 439; P. Li, E. Burdmann and R. Mehta, Nat. Rev. Nephrol., 2013, 9, 133.

2 N. Obermüller, H. Geiger, C. Weipert and A. Urbschat, Int. Urol. Nephrol., 2014, 46, 1; W. Prozialeck, J. Edwards, P. Lamar, J. Liu, V. Vaidya and J. Bonventre, Toxicol. Appl. Pharmacol., 2009, 238, 306; N. Hatipoğlu, O. Evliyaoğlu, B. Işxılk, M. Bodakçi, Y. Bozkurt, A. Sancaktutar, H. Söylemez, M. Atar, N. Penbegül, M. Yünce and 
M. Dağgulli, J. Endourol., 2014, 28, 224; B. Humphreys, F. Xu, V. Sabbisetti, I. Grgic, S. Naini, N. Wang, G. Chen, S. Xiao, D. Patel, J. Henderson, T. Ichimura, S. Mou, S. Soeung, A. McMahon, V. Kuchroo and J. Bonventre, J. Clin. Invest., 2013, 123, 4023.

3 B. Lane, Urol. Oncol.: Semin. Orig. Invest., 2013, 31, 682; V. Pennemans, L. De Winter, C. Faes, E. Kerkhove, C. Reynders, J. Rigo, Q. Swennen and J. Penders, Clin. Chim. Acta, 2010, 411, 2083; B. Dedeoglu, H. De Geus, G. Fortrie and M. Betjes, Biomarkers Med., 2013, 7, 947; X. Shao, L. Tian, W. Xu, Z. Zhang, C. Wang, C. Qi, Z. Ni and S. Mou, PLOS ONE, 2014, 9, e84131.

4 V. Vaidya, J. Ozer, F. Dieterle, F. Collings, V. Ramirez, S. Troth, N. Muniappa, D. Thudium, D. Gerhold, D. Holder, N. Bobadilla, E. Marrer, E. Perentes, A. Cordier, A. Vonderscher, G. Maurer, P. Goering, F. Sistare and J. Bonventre, Nat. Biotechnol., 2010, 28, 478; V. Vaidya, S. Waikar, M. Ferguson, F. Collings, K. Sunderland, C. Gioules, G. Bradwin, R. Matsouaka, R. Betensky, G. Curhan and J. Bonventre, Clin. Transl. Sci., 2008, 1, 200; R. Carson and D. Vignali, J. Immunol. Methods, 1999, 227, 41. 5 E. Randviir and C. Banks, Sens. Actuators, B, 2013, 183, 239; J. Metters, R. Kadara and C. Banks, Analyst, 2011, 136, 1067; U. Lad, S. Khokar and G. Kale, Anal. Chem., 2008, 80, 7910.

6 P. Kannan, H. Tiong and D. Kim, Biosens. Bioelectron., 2012, 31, 32.

7 Y. Huang, P. Hsiao and H. Chai, Ceram. Int., 2011, 37, 1825. 8 R. Chakraborty, S. Bepari and A. Banerjee, Bioresour. Technol., 2011, 102, 3610; S. Kongsri, K. Janpradit,
K. Buapa, S. Techawongstien and S. Chanthai, Chem. Eng. J., 2013, 215-216, 522.

9 Y. Ding, J. Liu, H. Wang, G. Shen and R. Yu, Biomaterials, 2007, 28, 2147; B. Wang, J. Zhang, Z. Pan, X. Tao and H. Wang, Biosens. Bioelectron., 2009, 24, 1141.

10 Y. Zhang, Y. Liu, X. Ji, C. Banks and W. Zhang, J. Mater. Chem., 2011, 21, 7552; Y. Zhang, Y. Liu, X. Ji, C. Banks and W. Zhang, J. Mater. Chem., 2011, 21, 14428; Y. Zhang, Y. Liu, X. Ji, C. Banks and W. Zhang, Chem. Commun., 2011, 47, 4126.

11 P. Kanchana, N. Lavanya and C. Sekar, Mater. Sci. Eng., C, 2014, 35, 85.

12 H. Zhao, X. Xu, J. Zhang, W. Zheng and Y. Zheng, Bioelectrochemistry, 2010, 78, 124.

13 Y. Dai, Y. Cai, Y. Zhao, D. Wu, B. Liu, R. Li, M. Yang, Q. Wei, B. Du and H. Li, Biosens. Bioelectron., 2011, 28, 112.

14 S. Zhu, J. Zhang, X. Zhao, H. Wang, G. Xu and J. You, Microchim. Acta, 2014, 181, 445; Q. Sheng, R. Liu and J. Zheng, Bioelectrochemistry, 2013, 94, 39; H. Yang, Z. Li, X. Wei, R. Huang, H. Qi, Q. Gao, C. Li and C. Zhang, Bioelectrochemistry, 2013, 94, 39; X. Jia, Z. Liu, N. Liu and Z. Ma, Biosens. Bioelectron., 2014, 53, 160.

15 T. Ikoma, H. Kobayashi, J. Tanaka, D. Walsh and S. Mann, J. Struct. Biol., 2003, 142, 327; F. Torres, O. Troncoso, J. Nakamatsu, C. Grande and C. Gómez, Mater. Sci. Eng., C, 2008, 28, 1276.

16 V. S. Vaidya, G. M. Ford, S. S. Waikar, Y. Wang, M. B. Clement, V. Ramirez, W. E. Glaab, S. P. Troth, F. D. Sistare, W. C. Prozialeck, J. R. Edwards, A. Bobadilla, S. C. Mefferd and J. V. Bonventre, Kidney Int., 2009, 76, 108. 